\title{
RESEARCHARTICLE
}

\section{SYNTHESIS, CHARACTERIZATION AND ANTIMICROBIAL ACTIVITY OF SOME NOVEL 1,3,4-OXADIAZOLE BASED DITHIAZA-SPIRONES}

\author{
Mahipal Reddy Yata $^{1}$, Ravinder Reddy Kunduru ${ }^{2}$, Srinivas Boche ${ }^{1}$ and Ravi Prasad Talagadadivi ${ }^{1 *}$ \\ ${ }^{1}$ Department of Chemistry, Kakatiya University, Warangal, Telangana, A.P.-506009, India \\ ${ }^{2}$ University College of Pharmaceutical Sciences, Kakatiya University, Warangal, Telangana-506009, India
}

*Corresponding author e-mail: drmrvr@gmail.com

\begin{abstract}
:
A novel series of 4-phenyl-9-(5-phenyl-[1,3,4-oxadiazol-2-yl)-1,6-dithia-4,9-diaza-spiro-[4.4]-nonane-3,8-diones (4a-e) in good to excellent yields by using commercially available 2 -amino-5-phenyl-1,3,4-oxadiazole (1) as raw material and 2-chloro$N$-(5-phenyl-[1,3,5]-oxadiazol-2-yl)-acetamide (2) and 2-benzylidene-3-(5-phenyl-[1,3,5]-oxadiazol-2-yl)-thiazolidin-4-onea (3a-e) as intermediates. The chemical structures of these compounds have been established by $\mathrm{IR},{ }^{1} \mathrm{H},{ }^{13} \mathrm{C}-\mathrm{NMR}$, Mass spectral data and elemental analysis. The newly synthesized compounds were screened for their ability towards antimicrobial activity.
\end{abstract}

Keywords: 1,3,4-oxadiazol derivatives, Chemical structure, antimicrobial activity

\section{INTRODUCTION}

1, 3, 4-Oxadiazoles are biologically active, synthetically useful and important heterocyclic compounds. Different classes of oxadiazole compounds possess an extensive spectrum of pharmacological activities such as antibacterial $^{1}$, antimalarial ${ }^{2}$, anti-inflammatory ${ }^{3}$, antifungal $^{4}, \quad$ anticonvulsant ${ }^{5}, \quad$ analgesic $^{6}$ antimycobacterial $^{7}, \quad$ antitumor $^{8}, \quad$ herbicidal $^{9}$, vasodialatory $^{10}, \quad$ cytotoxic $^{11}$, hypolipidemic ${ }^{12}$, ulcerogenic $^{13}$ and antiedema ${ }^{14}$. Numerous thiazolidinone derivatives have shown significant pharmacological and biological activities ${ }^{15}$ like sedative ${ }^{16}$, antiinflammatory $^{17}, \quad$ antibacterial $^{18}, \quad$ antifungal $^{19}$, $\operatorname{antitubercular}^{20}{ }^{\prime}$ anticancer $^{21}$, hypnotic ${ }^{22}$, anti-HIV ${ }^{23}$ and nematicidal $^{24}$.

\section{ANTIMICROBIAL ACTIVITY}

The in vitro antimicrobial activity of newly synthesized compounds, 4-phenyl-9-(5-phenyl-[1,3,4]-oxadiazol-2yl)-1,6-dithia-4,9-diaza-spiro-[4.4]-nonane-3,8-diones

(4a-e) was determined using disc diffusion method ${ }^{6}$. The antibacterial activity was measured against two grampositive strains viz., Staphylococcus aureus and Bacillus subtilis and two gram-negative strains viz., Escherichia coli and Pseudomonas aeruginosa and expressed in terms of zone of inhibition in $\mathrm{mm}$ at concentration of 5 $\mu \mathrm{g} /$ disc. The antifungal evaluation was carried out against fungal organisms namely Candida albicans and Aspergillus niger at concentration of $5 \mu \mathrm{g} /$ disc. Standard antibacterial drug Ciprofloxacin (5 $\mu \mathrm{g} /$ disc) and antifungal drug Fluconazole $(5 \mu \mathrm{g} /$ disc) were also tested under similar conditions against these organisms. Each experiment was done in triplicate and the average reading was taken.<smiles>[Y]N1C(=O)CSC12SCC(=O)N2c1nnc(-c2ccccc2)o1</smiles>

Scheme 1: 3, 4 Ar a ) = $\left.\left.\left.\left.\mathrm{C}_{6} \mathrm{H}_{5}, \mathrm{~b}\right)=4-\mathrm{CH}_{3}-\mathrm{C}_{6} \mathrm{H}_{4}, \mathrm{c}\right)=4-\mathrm{OCH}_{3}-\mathrm{C}_{6} \mathrm{H}_{4}, \mathrm{~d}\right)=4-\mathrm{Cl}-\mathrm{C}_{6} \mathrm{H}_{4}, \mathrm{e}\right)=4-\mathrm{Br}-\mathrm{C}_{6} \mathrm{H}_{4}$ 
The investigation of antibacterial screening data revealed that all the tested compounds exhibited significant and interesting biological activity, however with a degree of variation. According to the results (Table 1), it is clear that, some of the compounds displayed excellent antimicrobial activity. Among the series of the screened compounds 4a-e, the compound $\mathbf{4 e}$ which contain 4bromo phenyl ring system, against $E$. coli is highly active at $28 \mathrm{~mm}$ zone of inhibition which is equal to the standard drug Ciproflaxacin. The compound $4 \mathbf{e}$ towards
C. albicans is also exhibited good antifungal activity at $24 \mathrm{~mm}$ zone of inhibition which is almost equal to the standard drug. The remaining compounds showed moderate to good activity against the test organisms. It is interesting to note that, none of the compound is inactive against all the tested microorganisms and this remarkable property may achieve to the compounds due to the two active pharmacophores (oxadiazole and thiazole) in a single molecular skeleton.

Table 1 Antimicrobial activity of compounds 4a-e (Zone of inhibition in mm)

\begin{tabular}{lcccccc}
\hline Compound & \multicolumn{3}{c}{ Antibacterial activity } & \multicolumn{3}{c}{ Antifungal activity } \\
& S. aureus & B. subtilis & E. coli & P. aeruginosa & C. albicans & A. niger \\
\hline 4a & 12 & 20 & 13 & 19 & 12 & 12 \\
$\mathbf{4 b}$ & 11 & 18 & 10 & 14 & 13 & 10 \\
$\mathbf{4 c}$ & 14 & 12 & 18 & 12 & 15 & 16 \\
4d & 20 & 21 & 26 & 20 & 19 & 20 \\
4e & 24 & 23 & 28 & 20 & 24 & 22 \\
Ciprofloxacin & 26 & 26 & 28 & 25 & - & - \\
Fluconazole & - & - & - & - & 26 & 25 \\
\hline
\end{tabular}

\section{EXPERIMENTAL}

All reagents and solvents were used as purchased without further purification. Melting points were determined on a Fisher-Johns melting point apparatus and are uncorrected. Crude products were purified by column chromatography on silica gel of 60-120 mesh. IR spectra were obtained on a PerkinElmer BX series FT-IR 5000 spectrometer using KBr pellet. NMR spectra were recorded on a Varian $300 \mathrm{MHz}$ spectrometer for ${ }^{1} \mathrm{H}$ NMR. The chemical shifts were reported as ppm down field using TMS as an internal standard. Mass spectra were recorded on a VG-Micromass $7070 \mathrm{H}$ spectrometer operating at $70 \mathrm{eV}$.

Synthesis of 2-chloro- $N$-(5-phenyl-[1,3,5]-oxadiazol-2yl)-acetamide (2)

A mixture of 2-amino, 5-phenyl-1,3,4-oxadiazole (1) (0.01 mol) and chloroacetyl chloride (0.01 mol) was refluxed in triethyl amine $(10 \mathrm{ml})$ for $4 \mathrm{~h}$. After completion of the reaction (monitored by TLC), the resultant solution was cooled, the separated solid was filtered, dried and recrystallised from pet-ether to get pure 2-chloro-N-(5-phenyl-[1,3,5]-oxadiazol-2-yl)acetamide (2).

Synthesis of 2-benzylidene-3-(5-phenyl-[1,3,5]oxadiazol-2-yl)-thiazolidin-4-one (3)
Compound 2 (0.01 mol) was treated with phenyl isocyanate $(0.01 \mathrm{~mol})$ at room temperature in presence of $\mathrm{K}_{2} \mathrm{CO}_{3}(0.5 \mathrm{~g})$ in acetonitrile $(15 \mathrm{ml})$. The reaction mixture was refluxed on constant stirring for $6 \mathrm{~h}$. After conventional work up, the product was purified by recrystallization from methanol to give pure 2benzylidene-3-(5-phenyl-[1,3,5]-oxadiazol-2-yl)thiazolidin-4-one (3).

Synthesis of 4-phenyl-9-(5-phenyl-[1,3,4]-oxadiazol-2yl)-1,6-dithia-4,9-diaza-spiro-[4.4]-nonane-3,8-dione (4a-e)

An equimolar mixture of compound $3(0.01 \mathrm{~mol})$ and mercapto acetic acid $(0.01 \mathrm{~mol})$ was refluxed in dioxane for 7-9 h. After completion of the reaction (monitored by TLC), the reaction mixture was allowed to cool and was poured over crushed ice. The organic layer was extracted with ethyl acetate $(20 \mathrm{ml})$, washed with $10 \%$ sodium bicarbonate solution ( 1 X $20 \mathrm{ml})$ and dried with anhydrous sodium sulphate. The solvent was removed under vacuum and residue was recrystallized from methanol to yield the corresponding 4-phenyl-9-(5phenyl-[1,3,4]-oxadiazol-2-yl)-1,6-dithia-4,9-diazaspiro-[4.4]-nonane-3,8-dione (4a-e). 


\section{PHYSICAL AND SPECTRAL DATA}

\section{2-Chloro- $N$-(5-phenyl-[1,3,5]-oxadiazol-2-yl)-acetamide (2)}

White solid; Yield 74\%; Mp 155-157 ${ }^{\circ} \mathrm{C}$; IR (KBr) $3316(\mathrm{~N}-\mathrm{H}), 3024(\mathrm{C}-\mathrm{H}, \mathrm{Ar}), 2965(\mathrm{C}-\mathrm{H}), 1680(\mathrm{C}=\mathrm{O}), 1640(\mathrm{C}=\mathrm{N})$ $1560(\mathrm{C}=\mathrm{C}, \mathrm{Ar}) \mathrm{cm}^{-1} ;{ }^{1} \mathrm{H}$ NMR $\left(300 \mathrm{MHz}, \mathrm{CDCl}_{3}\right) \delta 4.21\left(\mathrm{~s}, 2 \mathrm{H}, \mathrm{CH}_{2}\right), 7.12-7.69(\mathrm{~m}, 5 \mathrm{H}, \mathrm{Ar}-\mathrm{H}), 7.16(\mathrm{~s}, 1 \mathrm{H}, \mathrm{NH}) ;{ }^{13} \mathrm{C}$ NMR $\left(100 \mathrm{MHz}, \mathrm{CDCl}_{3}\right) \delta 165.7,159.3,147.2,137.2,134.6,131.8$ (2), 125.7 (2), 45.8; MS m/z 237 (M $\mathrm{M}^{+}$); Elemental analysis: Calculated for $\mathrm{C}_{10} \mathrm{H}_{8} \mathrm{ClN}_{3} \mathrm{O}_{2}$ : C-50.54, H-3.39, Cl-14.92, N-17.68, O-13.47. Found: C-48.23, H-3.12, Cl$13.68, \mathrm{~N}-16.45, \mathrm{O}-12.78$.

\section{2-Benzylidene-3-(5-phenyl-[1,3,5]-oxadiazol-2-yl)-thiazolidin-4-one (3a)}

Yellow solid; Yield 72\%; Mp 163-165 ${ }^{0} \mathrm{C}$; IR (KBr) $3035(\mathrm{C}-\mathrm{H}, \mathrm{Ar}), 1690(\mathrm{C}=\mathrm{O}), 1630(\mathrm{C}=\mathrm{N}), 1568(\mathrm{C}=\mathrm{C}, \mathrm{Ar}) \mathrm{cm}^{-1}$; ${ }^{1} \mathrm{H}$ NMR $\left(300 \mathrm{MHz}, \mathrm{CDCl}_{3}\right) \delta 4.26\left(\mathrm{~s}, 2 \mathrm{H}, \mathrm{CH}_{2}\right), 6.98-7.89(\mathrm{~m}, 10 \mathrm{H}, \mathrm{Ar}-\mathrm{H}) ;{ }^{13} \mathrm{C} \mathrm{NMR}\left(100 \mathrm{MHz}, \mathrm{CDCl}_{3}\right) \delta 164.8$, 161.3, 160.4, 144.8, 136.1, 134.2, 132.8, 130.6 (2), 129.3 (2), 126.8 (2), 125.3, 124.7 (2), 38.1; MS m/z $336\left(\mathrm{M}^{+}\right)$; Elemental analysis: Calculated for $\mathrm{C}_{17} \mathrm{H}_{12} \mathrm{~N}_{4} \mathrm{O}_{2} \mathrm{~S}$ : C-60.70, H-3.60, N-16.66, O-9.51, S-9.53. Found: C-58.79, H-3.32, N-15.64, O-8.67, S-8.74.

\section{2-(4-Methyl-benzylidene)-3-(5-phenyl-[1,3,5]-oxadiazol-2-yl)-thiazolidin-4-one (3b)}

Pale yellow solid; Yield 76\%; Mp 132-134 ${ }^{\circ} \mathrm{C}$; IR (KBr) 3041 (C-H, Ar), $2945\left(\mathrm{C}-\mathrm{H}, \mathrm{CH}_{3}\right), 1685(\mathrm{C}=\mathrm{O}), 1642(\mathrm{C}=\mathrm{N})$, $1610(\mathrm{C}=\mathrm{C}) \mathrm{cm}^{-1} ;{ }^{1} \mathrm{H}$ NMR $\left(300 \mathrm{MHz}, \mathrm{CDCl}_{3}\right) \delta 2.43\left(\mathrm{~s}, 3 \mathrm{H}, \mathrm{CH}_{3}\right), 4.26\left(\mathrm{~s}, 2 \mathrm{H}, \mathrm{CH}_{2}\right), 7.01-7.87(\mathrm{~m}, 5 \mathrm{H}, \mathrm{Ar}-\mathrm{H}), 7.33$ $(\mathrm{d}, 2 \mathrm{H}, J=7.4 \mathrm{~Hz}, \mathrm{ArH}), 7.45(\mathrm{~d}, 2 \mathrm{H}, J=7.4 \mathrm{~Hz}, \mathrm{ArH}) ;{ }^{13} \mathrm{C} \mathrm{NMR}\left(100 \mathrm{MHz}, \mathrm{CDCl}_{3}\right) \delta 164.3,160.8,156.7,149.2$, 137.4, 135.3, 133.2, 132.7, 131.8 (2), 130.8, 129.4 (2), 126.7 (2), 124.9, 38.2, 22.6; MS m/z 350 ( $\mathrm{M}^{+}$); Elemental analysis: Calculated for $\mathrm{C}_{18} \mathrm{H}_{14} \mathrm{~N}_{4} \mathrm{O}_{2} \mathrm{~S}$ : C-61.70, H-4.03, N-15.99, O-9.13, S-9.15. Found: C-59.64, H-3.94, N-14.85, O8.69, S-8.74.

\section{2-(4-Methoxy-benzylidene)-3-(5-phenyl-[1,3,5]-oxadiazol-2-yl)-thiazolidin-4-one (3c)}

Orange solid; Yield 71\%; Mp 160-162 ${ }^{\circ} \mathrm{C}$; IR (KBr) $3038(\mathrm{C}-\mathrm{H}, \mathrm{Ar}), 2928\left(\mathrm{C}-\mathrm{H}, \mathrm{CH}_{3}\right), 1685(\mathrm{C}=\mathrm{O}), 1635(\mathrm{C}=\mathrm{N}), 1575$ $(\mathrm{C}=\mathrm{C}) \mathrm{cm}^{-1} ;{ }^{1} \mathrm{H}$ NMR $\left(300 \mathrm{MHz}, \mathrm{CDCl}_{3}\right) \delta 3.72\left(\mathrm{~s}, 3 \mathrm{H}, \mathrm{OCH}_{3}\right), 4.10\left(\mathrm{~s}, 2 \mathrm{H}, \mathrm{CH}_{2}\right), 7.15-7.69(\mathrm{~m}, 5 \mathrm{H}, \mathrm{Ar}-\mathrm{H}), 7.42(\mathrm{~d}$, $2 \mathrm{H}, J=7.2 \mathrm{~Hz}, \mathrm{ArH}), 7.54(\mathrm{~d}, 2 \mathrm{H}, J=7.2 \mathrm{~Hz}, \mathrm{ArH}) ;{ }^{13} \mathrm{C} \mathrm{NMR}\left(100 \mathrm{MHz}, \mathrm{CDCl}_{3}\right) \delta 163.2,161.8,158.6,155.3,151.7$, 146.4, 135.3, 134.2, 132.4, 130.7 (2), 128.6 (2), 125.8, 114.8 (2), 58.6, 23.7; MS m/z 366 (M ${ }^{+}$); Elemental analysis: Calculated for $\mathrm{C}_{18} \mathrm{H}_{14} \mathrm{~N}_{4} \mathrm{O}_{3} \mathrm{~S}$ : C-59.01, H-3.85, N-15.29, O-13.10, S-8.75. Found: C-57.62, H-3.27, N-14.39, O-12.86, S-7.95.

\section{2-(4-Chloro-benzylidene)-3-(5-phenyl-[1,3,5]-oxadiazol-2-yl)-thiazolidin-4-one (3d)}

Brown solid; Yield 73\%; Mp 150-152 ${ }^{0} \mathrm{C}$; IR (KBr) $3028(\mathrm{C}-\mathrm{H}, \mathrm{Ar}), 1695(\mathrm{C}=\mathrm{O}), 1630(\mathrm{C}=\mathrm{N}), 1584(\mathrm{C}=\mathrm{C}) \mathrm{cm}^{-1} ;{ }^{1} \mathrm{H}$ NMR (300 MHz, $\left.\mathrm{CDCl}_{3}\right) \delta 4.21\left(\mathrm{~s}, 2 \mathrm{H}, \mathrm{CH}_{2}\right), 7.09-7.54(\mathrm{~m}, 5 \mathrm{H}, \mathrm{Ar}-\mathrm{H}), 7.39(\mathrm{~d}, 2 \mathrm{H}, J=7.0 \mathrm{~Hz}, \mathrm{ArH}), 7.56(\mathrm{~d}, 2 \mathrm{H}, J=$ $7.0 \mathrm{~Hz}, \mathrm{ArH}) ;{ }^{13} \mathrm{C}$ NMR $\left(100 \mathrm{MHz}, \mathrm{CDCl}_{3}\right) \delta 163.5,160.8,158.7,151.0,143.7,133.8,131.2,130.7,129.4$ (2), 128.7 , 127.6 (2), 125.4 (2), 123.6, 35.7; MS m/z $370\left(\mathrm{M}^{+}\right)$; Elemental analysis: Calculated for $\mathrm{C}_{17} \mathrm{H}_{11} \mathrm{ClN}_{4} \mathrm{O}_{2} \mathrm{~S}: \mathrm{C}-55.06, \mathrm{H}-$ 2.99, Cl-9.56, N-15.11, O-8.63, S-8.65. Found: C-53.84, H-2.27, Cl-8.72, N-14.39, O-7.86, S-7.84.

\section{2-(4-Bromo-benzylidene)-3-(5-phenyl-[1,3,5]-oxadiazol-2-yl)-thiazolidin-4-one (3e)}

Pale yellow solid; Yield 74\%; Mp 144-146 ${ }^{\circ} \mathrm{C}$; IR (KBr) $3032(\mathrm{C}-\mathrm{H}, \mathrm{Ar}), 1680(\mathrm{C}=\mathrm{O}), 1631(\mathrm{C}=\mathrm{N}), 1578(\mathrm{C}=\mathrm{C}) \mathrm{cm}^{-1}$; ${ }^{1} \mathrm{H}$ NMR $\left(300 \mathrm{MHz}, \mathrm{CDCl}_{3}\right) \delta 3.98\left(\mathrm{~s}, 2 \mathrm{H}, \mathrm{CH}_{2}\right), 7.12-7.68(\mathrm{~m}, 5 \mathrm{H}, \mathrm{Ar}-\mathrm{H}), 7.42(\mathrm{~d}, 2 \mathrm{H}, J=7.4 \mathrm{~Hz}, \mathrm{ArH}), 7.74(\mathrm{~d}, 2 \mathrm{H}$, $J=7.4 \mathrm{~Hz}, \mathrm{ArH}) ;{ }^{13} \mathrm{C} \mathrm{NMR}\left(100 \mathrm{MHz}, \mathrm{CDCl}_{3}\right) \delta 167.7,161.6,159.6,156.7,149.2,135.3,1342,133.7,132.8(2)$, 131.7, 130.5 (2), 126.7 (2), 124.9, 38.2, MS m/z $415\left(\mathrm{M}^{+}\right)$; Elemental analysis: Calculated for $\mathrm{C}_{17} \mathrm{H}_{11} \mathrm{BrN}_{4} \mathrm{O}_{2} \mathrm{~S}: \mathrm{C}-49.17$, H-2.67, Br-19.24, N-13.49, O-7.71, S-7.72. Found: C-47.36, H-2.32, Br-17.46, N-12.39, O-6.94, S-6.84.

\section{4-Phenyl-9-(5-phenyl-[1,3,4]-oxadiazol-2-yl)-1,6-dithia-4,9-diaza-spiro-[4.4]-nonane-3,8-dione (4a)}

Brown solid; Yield 70\%; Mp 170-172 ${ }^{0} \mathrm{C}$; IR (KBr) 3048 (C-H, Ar), $1710(\mathrm{C}=\mathrm{O}), 1634(\mathrm{C}=\mathrm{N}), 1610$ (C=C, Ar), 1275 $(\mathrm{C}-\mathrm{S}) \mathrm{cm}^{-1} ;{ }^{1} \mathrm{H}$ NMR $\left(300 \mathrm{MHz}, \mathrm{CDCl}_{3}\right) \delta 4.18\left(\mathrm{~s}, 2 \mathrm{H}, \mathrm{CH}_{2}\right), 4.41\left(\mathrm{~s}, 2 \mathrm{H}, \mathrm{CH}_{2}\right), 7.12-7.69(\mathrm{~m}, 10 \mathrm{H}, \mathrm{Ar}-\mathrm{H}) ;{ }^{13} \mathrm{C} \mathrm{NMR}$ $\left(100 \mathrm{MHz}, \mathrm{CDCl}_{3}\right) \delta$ 167.5, 164.8, 153.2, 144.6, 141.6, 137.4, 131.8 (2), 129.7, 127.6 (2), 126.3 (2), $123.4,121.7$ (2), 76.4, 32.0, 28.6; MS m/z $410\left(\mathrm{M}^{+}\right)$; Elemental analysis: Calculated for $\mathrm{C}_{19} \mathrm{H}_{14} \mathrm{~N}_{4} \mathrm{O}_{3} \mathrm{~S}_{2}$ : C-55.60, H-3.44, N-13.65, O11.69, S-15.62. Found: C-54.36, H-3.14, N-12.95, O-10.74, S-14.82.

\section{4-(5-Phenyl-[1,3,4]-oxadiazol-2-yl)-9-p-tolyl-1,6-dithia-4,9-diaza-spiro-[4.4]-nonane-3,8-dione (4b)}

Yellow solid; Yield 77\%; Mp 140-142 ${ }^{\circ} \mathrm{C}$; IR (KBr) $3036(\mathrm{C}-\mathrm{H}, \mathrm{Ar}), 1710(\mathrm{C}=\mathrm{O}), 1634(\mathrm{C}=\mathrm{N}), 1623$ (C=C), 1285 (CS) $\mathrm{cm}^{-1} ;{ }^{1} \mathrm{H}$ NMR $\left(300 \mathrm{MHz}, \mathrm{CDCl}_{3}\right) \delta 2.39\left(\mathrm{~s}, 3 \mathrm{H}, \mathrm{CH}_{3}\right), 4.21\left(\mathrm{~s}, 2 \mathrm{H}, \mathrm{CH}_{2}\right), 4.28\left(\mathrm{~s}, 2 \mathrm{H}, \mathrm{CH}_{2}\right), 7.12-7.59(\mathrm{~m}, 5 \mathrm{H}, \mathrm{Ar}-$ $\mathrm{H}), 7.42(\mathrm{~d}, 2 \mathrm{H}, J=7.4 \mathrm{~Hz}, \mathrm{ArH}), 7.68(\mathrm{~d}, 2 \mathrm{H}, J=7.4 \mathrm{~Hz}, \mathrm{ArH}) ;{ }^{13} \mathrm{C} \mathrm{NMR}\left(100 \mathrm{MHz}, \mathrm{CDCl}_{3}\right) \delta 165.6,162.7,155.4$, 146.7, 143.0, 139.4, 134.2, 132.6 (2), 130.2, 127.6 (2), 125.8 (2), 120.9 (2), 74.8, 30.7, 26.4, 22.7; MS m/z $424\left(\mathrm{M}^{+}\right)$; Elemental analysis: Calculated for $\mathrm{C}_{20} \mathrm{H}_{16} \mathrm{~N}_{4} \mathrm{O}_{3} \mathrm{~S}_{2}$ : C-56.59, H-3.80, N-13.20, O-11.31, S-15.11. Found: C-54.95, H3.25, N-12.94, O-10.85, S-14.67.

\section{4-(4-Methoxy-phenyl)-9-(5-phenyl-[1,3,4]-oxadiazol-2-yl)-1,6-dithia-4,9-diaza-spiro-[4.4]-nonane-3,8-dione (4c)}

Orange solid; Yield 71\%; Mp 120-122 ${ }^{\circ} \mathrm{C}$; IR (KBr) 3038 (C-H, Ar), $1720(\mathrm{C}=\mathrm{O}), 1642(\mathrm{C}=\mathrm{N}), 1624(\mathrm{C}=\mathrm{C}), 1280(\mathrm{C}-$ $\mathrm{S}) \mathrm{cm}^{-1} ;{ }^{1} \mathrm{H}$ NMR $\left(300 \mathrm{MHz}, \mathrm{CDCl}_{3}\right) \delta 3.95\left(\mathrm{~s}, 3 \mathrm{H}, \mathrm{OCH}_{3}\right), 4.06\left(\mathrm{~s}, 2 \mathrm{H}, \mathrm{CH}_{2}\right), 4.19\left(\mathrm{~s}, 2 \mathrm{H}, \mathrm{CH}_{2}\right), 7.21-7.78(\mathrm{~m}, 5 \mathrm{H}, \mathrm{Ar}-$ (C) 2011-14, JDDT. All Rights Reserved 
$\mathrm{H}), 7.51(\mathrm{~d}, 2 \mathrm{H}, J=7.0 \mathrm{~Hz}, \mathrm{ArH}), 7.65(\mathrm{~d}, 2 \mathrm{H}, J=7.0 \mathrm{~Hz}, \mathrm{ArH}) ;{ }^{13} \mathrm{C} \mathrm{NMR}\left(100 \mathrm{MHz}, \mathrm{CDCl}_{3}\right) \delta 166.3,163.2,154.8$, 146.7, 143.7, 139.6, 136.7, 134.5 (2), 132.7 (2), 130.2, 128.7 (2), 123.4 (2), 78.7, 30.3, 26.3, 21.7; MS m/z $440\left(\mathrm{M}^{+}\right)$; Elemental analysis: Calculated for $\mathrm{C}_{20} \mathrm{H}_{16} \mathrm{~N}_{4} \mathrm{O}_{4} \mathrm{~S}_{2}$ : C-54.53, H-3.66, N-12.72, O-14.53, S-14.56. Found: C-52.68, H3.21, N-11.95, O-13.64, S-13.74.

\section{4-(4-Chloro-phenyl)-9-(5-phenyl-[1,3,4]-oxadiazol-2-yl)-1,6-dithia-4,9-diaza-spiro-[4.4]-nonane-3,8-dione (4d)}

Yellow solid; Yield 70\%; Mp 132-134 ${ }^{\circ} \mathrm{C}$; IR (KBr) 3051 (C-H, Ar), $1648(\mathrm{C}=\mathrm{N}), 1715(\mathrm{C}=\mathrm{O}), 1623(\mathrm{C}=\mathrm{C}), 1270(\mathrm{C}-$ S) $\mathrm{cm}^{-1} ;{ }^{1} \mathrm{H}$ NMR $\left(300 \mathrm{MHz}, \mathrm{CDCl}_{3}\right) \delta 4.18\left(\mathrm{~s}, 2 \mathrm{H}, \mathrm{CH}_{2}\right), 4.25\left(\mathrm{~s}, 2 \mathrm{H}, \mathrm{CH}_{2}\right), 7.12-7.59(\mathrm{~m}, 5 \mathrm{H}, \mathrm{Ar}-\mathrm{H}), 7.42(\mathrm{~d}, 2 \mathrm{H}, J=$ $7.8 \mathrm{~Hz}, \mathrm{ArH}), 7.65(\mathrm{~d}, 2 \mathrm{H}, J=7.8 \mathrm{~Hz}, \mathrm{ArH}) ;{ }^{13} \mathrm{C} \mathrm{NMR}\left(100 \mathrm{MHz}, \mathrm{CDCl}_{3}\right) \delta$ 166.3, 163.2, 151.7, 142.8, 138.7, 135.4, 131.7 (2), 129.6, 128.7 (2), 127.2, 125.3 (2), 119.8 (2), 73.7, 35.4, 29.7; MS m/z 444 (M ${ }^{+}$); Elemental analysis: Calculated for $\mathrm{C}_{19} \mathrm{H}_{13} \mathrm{ClN}_{4} \mathrm{O}_{3} \mathrm{~S}_{2}$ : C-51.29, H-2.95, Cl-7.97, N-12.59, O-10.79, S-14.41. Found: C-49.36, H-2.24, Cl6.98, N-11.94, O-9.94, S-13.75.

\section{4-(4-Bromo-phenyl)-9-(5-phenyl-[1,3,4]-oxadiazol-2-yl)-1,6-dithia-4,9-diaza-spiro-[4.4]-nonane-3,8-dione (4e)}

Orange solid; Yield 73\%; Mp 114-116 ${ }^{\circ} \mathrm{C}$; IR (KBr) $3048(\mathrm{C}-\mathrm{H}, \mathrm{Ar}), 1720(\mathrm{C}=\mathrm{O}), 1660(\mathrm{C}=\mathrm{N}), 1625(\mathrm{C}=\mathrm{C}), 1275(\mathrm{C}-$ S) $\mathrm{cm}^{-1} ;{ }^{1} \mathrm{H}$ NMR $\left(300 \mathrm{MHz}, \mathrm{CDCl}_{3}\right) \delta 4.15\left(\mathrm{~s}, 2 \mathrm{H}, \mathrm{CH}_{2}\right), 4.26\left(\mathrm{~s}, 2 \mathrm{H}, \mathrm{CH}_{2}\right), 7.18-7.58(\mathrm{~m}, 5 \mathrm{H}, \mathrm{Ar}-\mathrm{H}), 7.39(\mathrm{~d}, 2 \mathrm{H}, J=$ $7.8 \mathrm{~Hz}, \mathrm{ArH}), 7.68(\mathrm{~d}, 2 \mathrm{H}, J=7.8 \mathrm{~Hz}, \mathrm{ArH}) ;{ }^{13} \mathrm{C} \mathrm{NMR}\left(100 \mathrm{MHz}, \mathrm{CDCl}_{3}\right) \delta$ 162.7, 160.7, 151.8, 140.6, 138.9, 135.7, 132.8 (2), 129.8 (2), 126.8, 123.7 (2), 120.1 (2), 119.3, 78.7, 36.3, 30.2; MS m/z 487 (M ); Elemental analysis: Calculated for $\mathrm{C}_{19} \mathrm{H}_{13} \mathrm{BrN}_{4} \mathrm{O}_{3} \mathrm{~S}_{2}$ : C-46.63, H-2.68, Br-16.33, N-11.45, O-9.81, S-13.10. Found: C-44.39, H-2.31, Br$15.64, \mathrm{~N}-10.75$, O-9.28, S-12.46.

\section{RESULTS AND DISCUSSION}

The therapeutic importance of these rings prompted us to develop selective molecules in which a substituent could be arranged in a pharmacophoric pattern to display higher pharmacological activities. Thus we have designed and synthesized a series of novel 4-phenyl-9(5-phenyl-[1,3,4-oxadiazol-2-yl)-1,6-dithia-4,9-diazaspiro-[4.4]-nonane-3,8-dione (4) in good to excellent yields by using commercially available 2-amino-5phenyl-1,3,4-oxadiazole (1). The synthetic route leading to the title compounds is summarized in scheme 1.

The initial intermediate, 2-chloro- $N$-(5-phenyl-[1,3,5]oxadiazol-2-yl)-acetamide (2) was prepared through dehydrohalogenation between compound $\mathbf{1}$ and

\section{REFERENCES}

1. Andotra C S, Manhas B S, Acta Cienc. Indica Chem., 18, $1992,99$.

2. Hutt M P, Elstager E F, Werbet L M, J. Heterocycl. Chem., 7, 1970, 511

3. Silvestrini B, Pagatti C, Br. J. Pharmacol., 16, 1961, 209.

4. Sharma R S, Bahel S C, J. Indian Chem. Soc., 59, 1982, 877.

5. Omar A, Mohsen M E, Aboul Wafa O M, J. Heterocycl. Chem., 21, 1984, 1415.

6. Narayana B, Vijayaraj K K, Ashalatha B V, Kumari N S, Arch. Pharm., 2005, 338.

7. Ali M A, Yar M S, Bioorg. Med. Chem. Lett., 17, 2007, 3314.

8. Bezerra N M M, De-Oliveira S P, Srivastava R M, Da Silva J $\mathrm{R}$, Farmaco, 60, 2005, 955.

9. Ram V J, Pandey H N, Eur. J. Med. Chem., 25, 1990, 541.

10. Shirote P J, Bhatia M S, Arab. J. Chem., 2010, 145.

11. Padmavathi V, Reddy G S, Padmaja A, Kondaiah P, AliShazia. Eur. J. Med. Chem., 44, 2009, 2106.

12. Jayashankar B, Rai K M L, Baskaran N, Shazia H S S, Eur. J. Med. Chem., 44, 2009, 3898.

13. Shashikan D, Bhandari V, Bothara K G, Raut M K, Patil A A, Sarkate A P, Mokale V J, Bioorg. Med. Chem. Lett., 16, 2008, 1822 . chloroacetyl chloride in triethyl amine under reflux for 4 h. Compound $\mathbf{2}$ on cyclization with various phenyl thioisocyanates in presence of potassium carbonate in refluxing acetonitrile for $6 \mathrm{~h}$ to get the corresponding intermediate, 2-benzylidene-3-(5-phenyl-[1,3,5]oxadiazol-2-yl)-thiazolidin-4-one (3a-e). The title compounds 4-phenyl-9-(5-phenyl-[1,3,4-oxadiazol-2-yl)1,6-dithia-4,9-diaza-spiro-[4.4]-nonane-3,8-diones (4a-e) have been prepared from the subsequent ring closure reaction of compound $\mathbf{3}$ with mercapto acetic acid in refluxing 1,4-diaxane for 7-9 h. The chemical structures of all the newly synthesized compounds were confirmed by their IR, ${ }^{1} \mathrm{H},{ }^{13} \mathrm{C} \mathrm{NMR}$, mass spectral data and elemental analysis. Further the compounds 4a-e were used to evaluate their antimicrobial activity.

14. Omar F A, Mahfouz N M, Rahman M A, Eur. J. Med. Chem., 31, 1996, 819

15. Dave C V, Shukla M C, Indian J. Chem., 39B, 2000, 210.

16. Doran W J, Shoule H A, J. Org. Chem., 3, 1939, 193.

17. Menozzi G, Filippelli W, Farmaco, 49, 1994, 115

18. Barot V M, Asian J. Chem., 8, 1996, 802.

19. Khan M H, Nizamuddin A, J. Food Agric. Chem., 43, 1995, 2719.

20. Gangiee A, Abaer G, J. Med. Chem., 42, 1999, 2447.

21. Shah B R, Desai N C, Trivedi P B, Ind. J. Heter. Chem., 2, 1993, 249

22. Rahman A, El-Gazzar B A, Hafex H N, Acta. Chem. Slov., $55,2008,359$

23. Shah B R, Desai N, Trivedi P, Ind. J. Heter. Chem., 2, 1993, 249

24. Manrao M R, Monika J, Kaul V K, Pl. Dis. Res., 12, 1997, 70 .

25. National Committee for Clinical Laboratory Standards (NCCLS), Nat. Comm. Lab. Stands., Villanova, 1982, 242. 